\title{
基于传感设备和在线平台的自反馈式城市设计方法 及其实践
}

\author{
Methodology and Application of the Self-feedback Urban Design Based on Urban Sensors and Online Platform
}

摘要: 大数据的兴起, 深化了人们在城市规划与设计过程中对现状的精细化认识。 城市传感器基础设施和智慧城市的建设, 为规划设计的后置式反馈和精确描绘分析 对象提供了契机。在线平台不仅动态展示城市数据信息, 更激发了城市人群个体的 空间应变, 提供了空间需求信息反馈的渠道, 促进了不同角色的交互与沟通。本文 提出基于传感设备和在线平台的自反馈式城市设计方法, 在搭建整体框架的基础上, 解析数据和传感器类型, 划分在线平台的模块, 提炼五类应用场景。文章进一步将 这一自反馈式城市设计的方法初步应用于上海衡复历史街区的城市设计实践, 提出 了空间传感器植入框架, 结合多种大数据和开放数据, 形成融合多种功能模块的衡 复区信息共享网络交互平台, 包括街道慢行指数测度平台、人本观测平台、人迹地 图平台、方案宣传展示平台、公众参与平台、人际数据交互感知地毯数据平台等六 大版块, 并对其所带来的机遇和挑战进行了讨论。

Abstract: The burst of urban big data has deepened people's perception of urban context. However, the construction of smart-city infrastructure and urban sensors implies the opportunity of delicate depiction of existing situation and post-positioned feedback of the implementation of planning and design. The online platform not only vividly displays various city information, but also creates the channel for feedback of new demands of urban life, and facilitates the interaction of the roles and stakeholders. This paper proposes the framework of self-feedback urban design based on urban sensors and online platform. Firstly, it introduces the workflow, data inventory, urban sensor category, online platform modules and application scenarios. Secondly, this methodology is applied to urban design of Hengfu Historical District in Shanghai, with a comprehensive online platform combining the modules of slow-traffic data collection and analysis platform, human observational data platform, human activity track platform, design and project display platform, public participation platform, spatial interaction carpet platform. At last, the opportunities and challenges of this self-feedback urban design methodology are further discussed.

关键词: 数据增强设计; 城市传感器; 在线平台; 空间干预;

$$
\text { 上海衡复历史街区 }
$$

Keywords: Data Augmented Urban Design; Urban Sensor; Online Platform; Spatial Intervention; Hengfu Historical District in Shanghai “清华大学(建筑学院) 一旭辉控股 (集团) 有限公司可持续住区联合研究中心”
课题经费支持

作者: 龙瀛, 清华大学建筑学院、清华大学恒隆房地产研究中心, 副教授, 博士生导师 曹哲静 (通信作者), 清华大学建筑学院, 博士研究生

\section{引言}

大数据的兴起, 深化了人们在城市规划与 设计过程中对现状的精细化认识; 同时在现状研 究、方案生成、设计表现等环节形成了数据支撑 的技术体系。龙瀛和沈尧提出数据增强设计（data augmented design) 的规划设计新方法论 ${ }^{[1]}$, 旨在 利用 “以定量城市分析为驱动的规划设计方法, 通过数据分析、建模、预测等手段, 为规划设计 的全过程提供调研、分析、方案设计、评价、追 踪等支持工具, 以数据实证提高设计的科学性, 并激发规划设计人员的创造力” ${ }^{[2]}$ 。数据增强设计 继承了规划支持系统和决策支持系统, 针对增量 型规划设计, 可提取已有案例城市空间维度指标, 形成量化的城市空间维度的分类基因库和对新城 方案的借鉴指标系统 ${ }^{[3]}$; 针对存量型规划设计, 可 通过多维数据的现状分析提取问题, 并通过定量 评估衡量空间方案改造后的实施状况 ${ }^{[4]}$ 。

目前众多国际研究机构和企业从多个方面对 大数据在城市规划与设计中的运用进行了实例探 索（表 1)。在将以往碎片化和不可感知的空间信 息进行精细化度量和可视化的基础上, 主要形成 了五个方向的应用：一是通过搜集空间物质环境 数据分析现状的问题, 从而提出空间环境改进策 略; 二是通过分析人群对不同空间环境的使用状 态, 分析和发现其需求, 从而优化空间设施配置; 三是提出数理城市模型, 利用数据进行验证, 从 而预测未来的城市发展或评估规划设计方案; 四 是利用数据研究空间环境和行为的关系, 从而评 价不同设计方案的环境效应; 五是通过虚拟现实 
（virtual reality）和增强现实（augmented reality）的技术对未 来规划设计方案进行可视化模拟。国内不少学者响应 “数据 增强设计”的理念, 展开了研究和探索 : 盛强将空间句法分 析运用到设计教学中 ${ }^{[5]}$; 徐磊青等通过调研与定量分析研究 轨道交通站点对人流活动的影响, 并提出城市设计策略 ${ }^{[6]}$; 茅明睿等利用多种开放数据和大数据对回龙观地区的空间品 质与活力进行量化研究, 提出针对性的解决策略, 并搭建了 数据增强设计平台 ${ }^{[7,8]}$; 杨俊宴和曹俊提出大数据在城市设 计中的四种应用模式 ${ }^{[9]}$; 龙瀛和叶宇提出人本尺度的城市形 态测度、评估及规划设计相应框架 ${ }^{[10]}$ 。然而目前在大多数的 案例运用中, 大数据主要通过前置性反馈辅助现状分析和设 计方案的生成, 对于方案实施的后置性反馈和动态调整涉及 较少。此外尽管近年来规划设计领域产生了不少时空行为数 据, 但存在 “开放数据不大、大数据不开放” 等问题 ${ }^{[8]}$, 片 段性、稀疏、有偏的样本数据难以应对数据覆盖广度、时间 跨度、对象精度的问题 ${ }^{[11]}$ 。

城市传感器基础设施和智慧城市的建设不断兴起, 如芝 加哥城市运算和数据中心的 “物联城市” (Array of Things) 项目通过搭建城市传感器网络, 为居民、城市管理者和 科学家提供认识、分析和改造城市的数据基础（http:// arrayofthings.github.io/) ; 哥伦比亚大学的智慧城市研究中心 （http://datascience.columbia.edu/smart-cities）构建了智慧社 区的传感器系统, 提出营造社区安全性的方案; 麻省理工学 院（MIT）市民数据设计实验室通过开发新型传感器, 测量 公共空间中的人群感知与行为。人们通过自定义城市传感器 的类型、安装场地、监控时间和反馈周期, 获得了城市特定 区域空间要素或人群的详细数据, 并精准刻画其特征。这为 规划设计的后置式反馈和精确描绘分析对象提供了契机: 在 方案实施过程中, 通过自定义传感器的精准分析和短周期反 馈, 动态评估修正方案, 避免了蓝图式规划设计的失误。此外, 虚拟现实、增强现实和混合现实技术的发展引发了第四次科 技革命, 城市的物质空间逐渐依赖于虚拟网络来强化彼此的 链接, 人们对城市的主观感知不再直于传统物质空间的体验, 倾向于从网络空间获得更多新的认知与发现 ${ }^{[12]}$, 进而引发对 城市空间新的需求。整合多样化城市数据信息且可视化的开 放在线平台将成为重要的载体, 使得以往模糊的、碎片化的 城市信息被感知体验。个体在动态的感知下将形成新的行为 方式和空间需求, 继而将映射到真实空间的改造中。在线平

\section{表 1 国际典型研究机构和企业数据增强设计案例}

\begin{tabular}{|c|c|}
\hline 典型研究机构 & 代表性的研究项目 \\
\hline $\begin{array}{l}\text { 新加坡未来城市实验室（Future City Laboratory）（http://www.fcl. } \\
\text { ethz.ch/research/responsive-cities/big-data-informed-urban-design.html） }\end{array}$ & $\begin{array}{l}\text { 大数据城市设计项目 (Big Data Informed Urban Design) : 通过建立城市大数据分析框架形成交 } \\
\text { 互性的规划设计支持系统, 包括城市现状精细化分析、空间环境和行为关系研究、不同设计 } \\
\text { 方案的评价等 }\end{array}$ \\
\hline $\begin{array}{l}\text { 美国麻省理工学院市民数据设计实验室 } \\
\text { (MIT Civic Data Design Lab) (http://civicdatadesignlab.mit.edu/) }\end{array}$ & $\begin{array}{l}\text { (1) 奈落比市巴士数据项目 (Digital Matatus) : 通过开发手机 APP 得到众包的奈落比市巴士 } \\
\text { 轨迹数据, 优化巴士线路方案; } \\
\text { (2) 市民数据管理项目 (Civic Data Management) : 分析市民投诉电话信息的位置数据, 分析 } \\
\text { 其空间模式和影响因素, 优化规划措施 }\end{array}$ \\
\hline $\begin{array}{l}\text { 美国哥伦比亚大学城市规划空间信息实验室 } \\
\text { (Columbia University Urban Planning Spatial Information Lab) } \\
\text { (http://c4sr.columbia.edu/projects) }\end{array}$ & $\begin{array}{l}\text { (1) 利用大数据构建纽约健康城市分析网格; } \\
\text { (2) 分析纽约公共自行车的轨迹数据, 优化投放站点设计 }\end{array}$ \\
\hline $\begin{array}{l}\text { 新加坡一美国麻省理工学院可感知城市实验室（Senseable Cities } \\
\text { Lab Singapore-MIT Program）(http://senseable.mit.edu/） }\end{array}$ & $\begin{array}{l}\text { (1) 地下城市项目 (Underworld) : 通过城市下水系统环境数据分析城市公共卫生状况, 支撑 } \\
\text { 健康城市规划措施; } \\
\text { (2) 出租车数据分析项目 (Hubcab) : 通过分析纽约出租车数据, 提出道路共享设计方案 ; } \\
\text { (3) 城市感知项目 (Sense and the City) : 通过获取多源数据记录柏林市公共空间一日 } 24 \text { 小时 } \\
\text { 建成环境和人群活动数据, 分析空间使用状况 }\end{array}$ \\
\hline $\begin{array}{l}\text { 美国哈佛大学大数据与智慧城市中心 (Harvard Data-Smart City } \\
\text { Solutions)（http://datasmart.ash.harvard.edu/) }\end{array}$ & 分析城市基础设施和交通、健康、公共安全数据, 提供多样城市规划优化措施 \\
\hline $\begin{array}{l}\text { 日本东京大学空间信息科学中心 } \\
\text { (http://www.csis.u-tokyo.ac.jp/english/) }\end{array}$ & 基于大数据研发各类城市模型, 分析城市空间信息并优化规划设计决策 \\
\hline $\begin{array}{l}\text { 英国剑桥大学马丁中心 (Martin Centre, U Cambridge) } \\
\text { (https://www.martincentre.arct.cam.ac.uk/) }\end{array}$ & $\begin{array}{l}\text { (1) 基于大数据建立土地利用和交通一体化模型, 进行规划设计方案选择和评价 ; } \\
\text { (2) 基于大数据建立建筑和基础设施供需分析模型, 应用于英国南部城市的案例分析 }\end{array}$ \\
\hline $\begin{array}{l}\text { 美国加州大学伯克利分校城市模拟实验室 } \\
\text { (http://www.urbansim.com/) }\end{array}$ & $\begin{array}{l}\text { 城市与交通一体化模拟项目 (Urbansim)：利用多源数据通过建立土地利用和交通一体化模型, } \\
\text { 支持规划与设计决策 }\end{array}$ \\
\hline $\begin{array}{l}\text { 英国牛津互联网中心 (OII Oxford Internet Institute) } \\
\text { (https://www.oii.ox.ac.uk/) }\end{array}$ & 基于城市社交网络信息大数据, 提供规划设计决策基础 \\
\hline $\begin{array}{l}\text { 澳洲昆士兰科技大学城市信息中心 (QUT Urban Informatics } \\
\text { Centre) (https://www.urbaninformatics.net/) }\end{array}$ & $\begin{array}{l}\text { 增强城市模型项目（Augmented City Model）：运用增强现实（Augmented Reality）技术对城市 } \\
\text { 建成环境和人流数据进行可视化和分析, 或对规划设方案进行模拟 }\end{array}$ \\
\hline
\end{tabular}


台不仅是城市数据信息的动态展示, 更激发了城市人群个体 的空间应变, 提供了空间需求信息反馈的渠道, 促进了不同 角色的交互与沟通。

在此背景下, 本文提出了基于传感设备和在线平台的自 反馈式城市设计方法。该方法倡导通过在实体空间中布设能够 捕获人对空间使用状态和空间状态其变化的多元传感器, 在 虚拟空间建立在线平台以读取传感器、互联网和政府的多源

（准）实时数据；将后置式空间测度反馈与规划设计过程进行 结合, 将长周期的规划设计评估转换为短周期的空间反馈与空 间干预; 并在未来的城市建设中落实数据测度基础设施的建设, 通过精细化的 “订制大数据” 的反馈来实现设计方案和空间 使用的可持续良性互动, 进而实现对设计方案在整个场地内的 不同时间周期的实施效果进行监测和评价, 提出对设计方案的 调整与优化建议, 最终实现自反馈式的城市设计。笔者将这一 自反馈式城市设计的方法初步应用于上海衡复历史街区的城 市设计实践, 并对其所带来的机遇和挑战进行讨论。

\section{1 方法}

\section{1 整体框架}

基于传感器和在线平台的自反馈城市设计, 首先需要针 对特定的城市地区布置传感器设备, 明确其种类、数量和位 置; 其次选择合理的测度指标、测度时长与周期, 对设计方 案实施进行针对性的指标数据搜集; 再次通过在线平台设计 整合传感器数据、大数据、开放数据、政府数据等多源数据, 一是提炼关键指标对公众进行可视化的展示, 二是城市管理 者基于数据分析和指标度量对城市问题进行诊断并对规划方 案实施进行评估, 三是提供民众对规划方案实施等的意见反 馈渠道, 形成多元利益相关者在线沟通的平台。图 1 显示了 该方法的整体技术框架。与传统的前置式城市设计相比, 基 于传感器和在线平台的自反馈城市设计表现出数据动态更新 和及时反馈的特点 ${ }^{[13,14]}$ 。其反馈周期分为短周期反馈和实时 反馈两类。短周期反馈需将数据进行清洗、整理后提取有效 的分析信息, 用于指示某类环境和空间人群使用状态指标, 为规划设计重新干预、修正、调整提供基础参照。实时反馈 主要依托于设计好的程序与软件, 通过前期条件设定, 搜集 分析人群空间使用状态实时数据 ${ }^{[15]}$, 即时诱导人群行为、调 整空间设施分配。
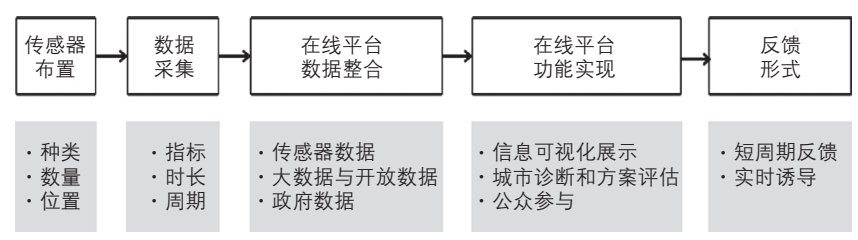

图 1 整体技术框架

\section{2 数据类型及传感器}

在线平台的数据源主要为开放数据、大数据和自定义的 传感器数据三类 (表 2)。开放数据主要为带有地理位置信 息的空间环境数据; 大数据主要为人类行为数据, 包括轨迹、 社交、活动和能耗等; 传感器数据与前两者的区别在于其自 定义和高精度的特点, 记录对象主要为与建成环境空间发生 关系的浮动人群和车辆等, 以及由于人群活动带来的环境变 化数据。传感器类型包括 WIFI 探针、GPS、摄像头和图像 采集器、人流卡口设施、环境声光热测度传感器、针对人群 行为诱导和空间设施调度的 APP 等。数据类型包括人群属性 数据 (人群画像) 、场地内即时人流量与轨迹数据、人群与 环境的交互数据等。

\section{3 在线平台}

平台的功能在于整合单一维度的数据源, 形成融合的分 析, 以不同功能版块呈现分析结果 ${ }^{[16]}$, 从而为民众、规划师、 城市管理者、开发商等提供信息参考、反馈渠道和协商平台。 图 2 显示了平台的整体架构, 分为 “数据获取一数据存储一 数据预处理一数据处理一数据分析一数据输出和展示” 六个 步骤。数据获取依赖于开放数据与大数据的接口, 以及传感 器搜集的数据, 可通过云端服务器和本地服务器进行存储。 在经过数据清洗和分类后, 可从时间尺度、空间尺度、人群 对象尺度三个维度进行数据处理。数据分析包括以上三个维 度不同的空间环境分析及人群活动分析两类, 最终形成数据 输出和展示, 包括空间要素在各个地理空间尺度的分布、空 间要素价格分布、空间环境品质衡量、空间中人群特征的分 布、人群对城市空间要素的满意度和可能需求等。

\section{4 应用场景}

基于开放数据、大数据、自定义传感器数据的城市设计 在线平台具有以下应用场景。一是监测设计方案实施状况并

\section{表 2 基于传感器和在线平台的城市设计所整合的三种数据类型}

城市形态数据（POI、路网、建筑三维形态等）; 城市用地数据（用 地性质和使用强度); 街景地图数据; 城市环境数据（空气污染、水

开放

数据

问数据（景点访问时段、微博签到地点与时间）; 点评数据（微博、 大众点评、旅游网站等）

大数据建筑能耗数据; 出行轨迹和交通 $\mathrm{OD}$ 数据 (公交、地铁、出租、自行车、 其他共享交通工具) ; 基于手机 APP 的人群画像数据

基于场地 WIFI 探针的人群画像 ; 基于人脸摄像头的人群画像 ; 场地

自定义 卡口人流数据; 建筑或构筑物等人群交互设施数据（人流量、声音

传感器 分贝等); 环境测度数据（噪音、PM2.5、日照、通风等）; 图像采集

数据 数据 (街景、人脸、机动车) ; 人群实时服务和诱导 APP (停车诱导 软件、游览驾车路线推荐软件、场地人流监控和疏散软件等) 数据 
进行空间修正, 主要针对于长期实施的设计方案, 对象通常 为城市重要的轴线廊道和节点地区。通过设定设计方案目标 实现的参数, 利用在线平台监测空间使用状况, 可对偏离基 础指标的空间要素进行干预修正和实施周期调整。二是基于 多情景预测的设计导则监测空间的变化, 进行相应的空间动 态干预, 主要针对于中短周期实施的设计方案, 对象通常为 城市次要的街道、节点和片区。通过预测提出多种情境下的 设计方案，设定情景发生变化的空间测度阈值，利用在线平 台周期性监控空间状况, 当超过情景变化的阈值时进行设计 方案的动态调整, 从而优化空间的使用状态。三是促进公众 参与, 即通过搜集城市设计覆盖人群于在线平台公开信息窗 口的反馈评价, 补充公众在方案实施阶段的参与过程。四是 城市设计实施的动态管理和紧急情况应对 ${ }^{[17]}$, 如通过舆情和 网络 APP 评价数据, 及时对城市消极空间进行干预管理 ; 利 用人流和车流数据监控公共空间拥堵的发生 ; 利用空间环境
声光热传感器监控灾害和险情的发生。五是通过空间与人群 交互数据的可视化增加空间的趣味性和启示性, 如结合建筑 与场地的交互装置对人群属性或流量进行动态可视化显示。 六是对人群行为进行实时诱导并优化空间设施配置, 如人们 可通过在线平台查询热门地点人群流量、停车位等信息以决 定最优出行计划; 政府通过对访问集中区域进行停车位和道 路使用费用调节, 实现交通实时诱导; 企业通过平台查询人 群绿色出行需求, 从而优化城市共享单车的空间资源配置等。 图 3 显示了以上六种场景在线平台的实现流程。

\section{2 应用案例}

本文以上海衡复历史街区慢行系统设计为例, 探讨基于 传感设备和在线平台的自反馈式城市设计方法的运用 ${ }^{1}$, 图 4 展示了其整体框架。方案不同于蓝图式的规划设计方案, 而 是基于多元开放数据和大数据的现状分析, 提出规划引导措

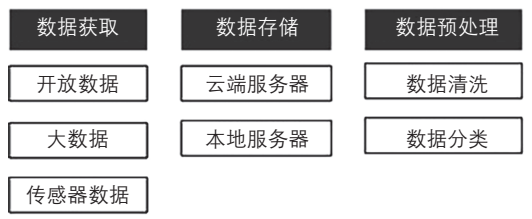

图 2 在线平台构架

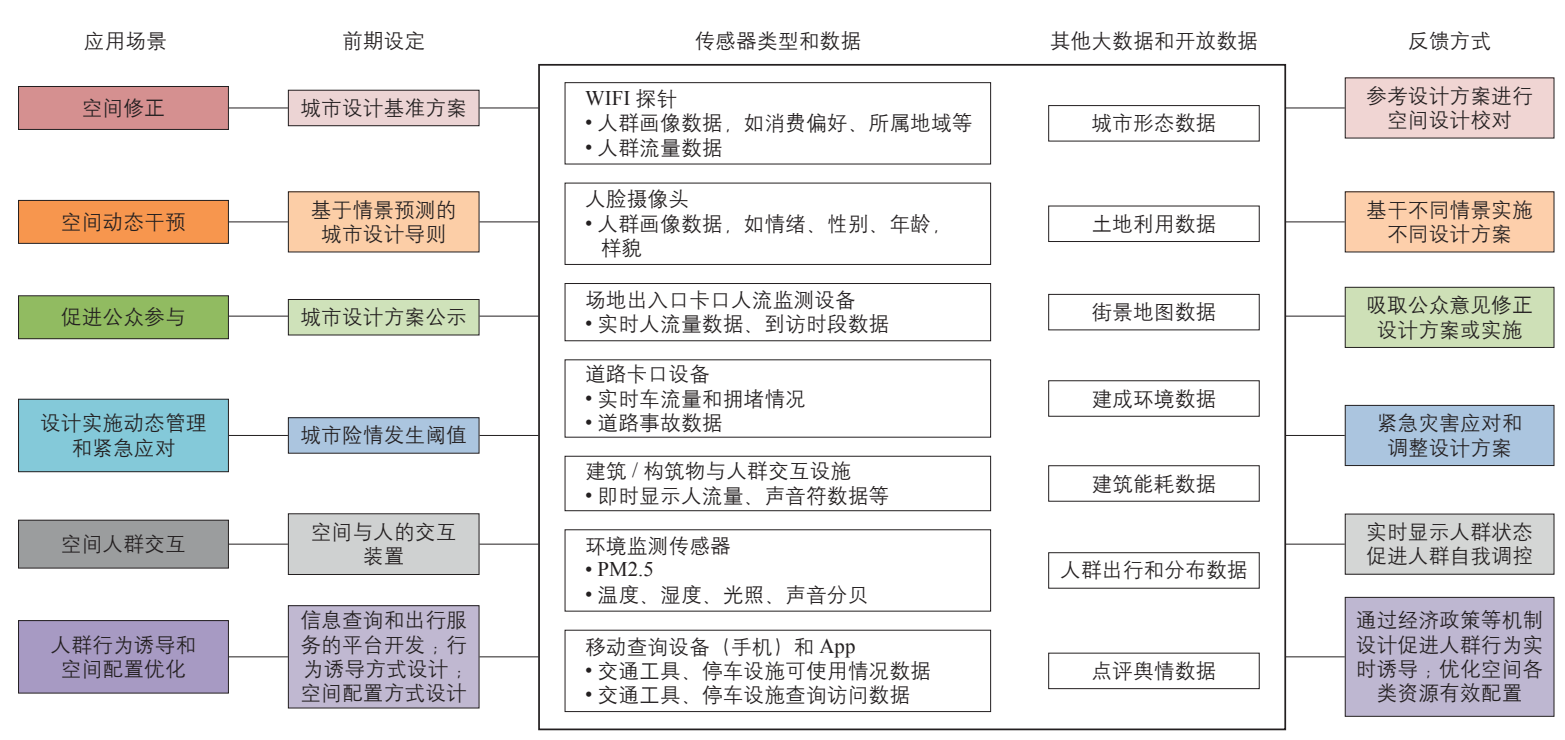

图 3 基于大数据、开放数据和传感器数据的在线平台在城市设计与实施中的应用场景

\section{数据分析}
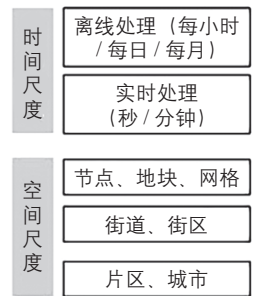

$人$
群
对
象

群

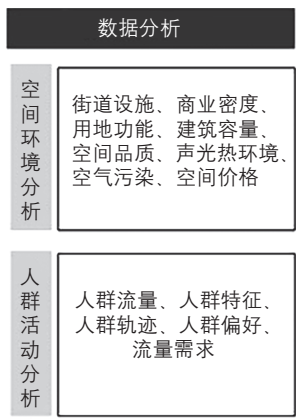

数据输出和展示

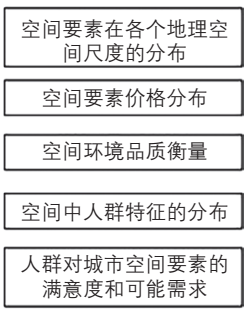

某一属性特征组群

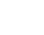


施, 并根据四个维度的街道慢行指数进行评分, 将现状街道 划分为维持并优化现有特征的 3 种 $\mathrm{A}$ 类街道和根据数据测度 动态调整的 4 种 $\mathrm{B}$ 类街道, 针对两类街道提出了不同的设计 导则与空间干预措施。为了对街道特征进行测度从而实现空 间修正、动态规划和促进公众参与, 本方案特提出了空间传 感器植人框架, 结合多种大数据和开放数据, 形成融合多种 功能模块的衡复区信息共享网络交互平台, 包括街道慢行指 数测度平台、人本观测平台、人迹地图平台、方案宣传展示 平台、公众参与平台、人际数据交互感知地毯数据平台等六 大版块 ${ }^{1}$ 。在明确不同指标数据搜集方式和搜集周期的基础上, 该方案主要针对 $\mathrm{A} / \mathrm{B}$ 类街道导则的实施状况、 $\mathrm{A} / \mathrm{B}$ 类街道是 否需要重新划分、交互装置的实时反馈、城市监控管理与调度、 信息传达与公众参与等五个维度进行城市设计的自反馈。

单一维度的反馈数据需要进行整合分析, 抽取有效的空 间测度指标抓手, 最终使得测度指标和不同的空间干预手段 相呼应。如数据的反馈周期和测度内容应对应空间干预的重 要层次和类型。其中 $\mathrm{A} / \mathrm{B}$ 类街道导则的实施状况为短周期 (以一季度至一年为单位) 的自反馈, 以街道的吸引力、安 全性、舒适性和历史性指标为抓手: A 类街道以中长期设计 方案为参照, 通过空间测度衡量规划设计方案的实施满意度, 从而不断修正空间改造状态; B 类街道基于情景预测的设计 导则对其动态引导, 空间测度用于衡量不同情景的发生状况, 从而适应性地选择设计导则工具进行空间动态调整。 $\mathrm{A} / \mathrm{B}$ 类 街道是否重新划分为中等周期 (以 1 5 年为单位) 的自反 馈, 通过测度街道整体步行指数、土地利用、设施分布、网 络與情、交通出行、土地经济要素、上位规划要求和市民反 馈等指标, 调整衡复历史保护街区慢行系统整体策略。此外 一些人群和空间交互装置需要进行实时数据反馈; 城市管理
者需要对重点地段进行人群活动的监控分析与管理调度 ; 城 市不同利益相关者可以通过规划设计方案展示和公众参与平 台传递自己的声音。

\section{1 街道慢行指数测度平台}

方案针对衡复历史街区构建了涵盖 20 个指标的街道慢 行指数平台 (图 5), 分为吸引力、安全性、舒适性和历史 性等四个维度。主要目的为根据 $\mathrm{A} / \mathrm{B}$ 类街道的更新周期提 取所需要的指标抓手和分析数据, 测度 $\mathrm{A} / \mathrm{B}$ 类街道设计导 则的实施状况。表 3 对 20 个指标的数据接口与数据分析进 行了总结。慢行指数测度对象为线性的街道空间, 数据更新 周期为半年左右, 其中针对街景图片评分的部分未来可通过 机器学习自动批量完成。

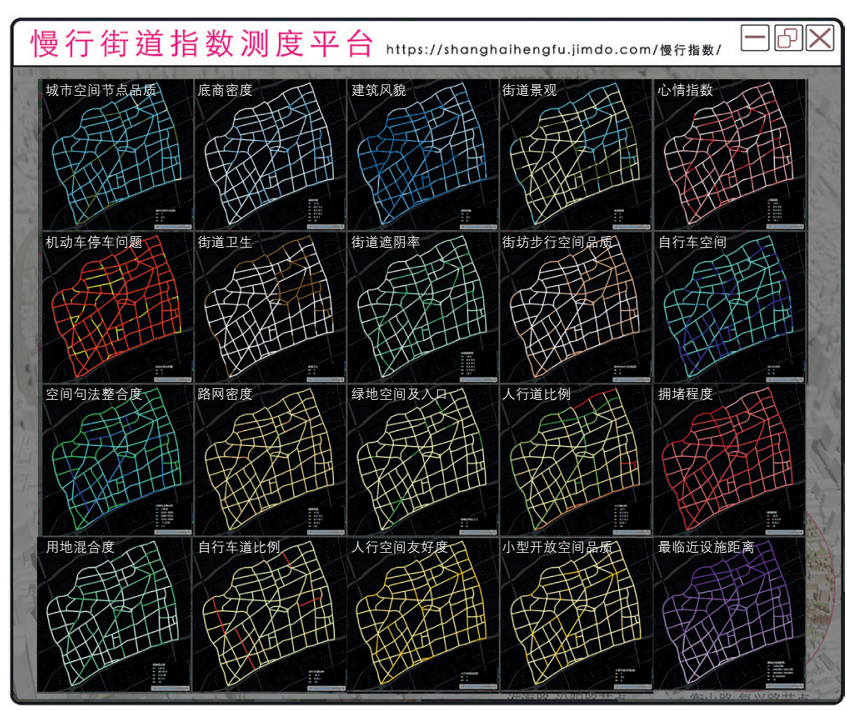

图 5 衡复历史街区街道慢行指数测度平台
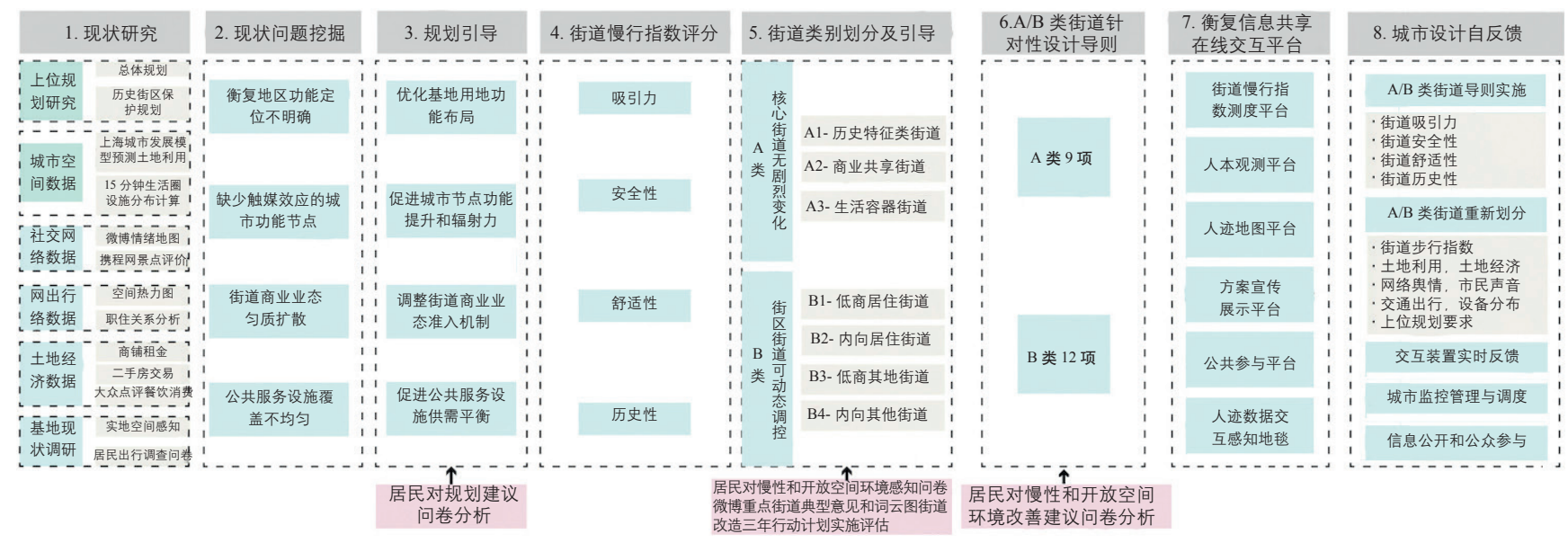

\section{图 4 衡复历史街区慢行系统设计整体框架}

(1) 该方案获得2016年上海城市设计挑战赛衡复专业赛区的第二名。 


\section{2 人本观测平台}

衡复人本观测平台（图 6）是基于街区尺度的人群观测平 台, 旨在从微观尺度观测重点街道的人流、與情以及空间变化, 并实现空间可视化。表 4 显示了具体的功能模块、数据接口 和传感器类型。其主要目的是短期（天、星期、月）使得城 市管理者对城市空间进行观测、管理与调度; 长期（1 年左右） 实现数据采集和应用, 辅助追踪街道设计方案的实施效果, 了 解居民的意见。最理想的情况是分析模块可以配合街道设计的 不断更新完善，周期性地提供观测结果和意见反馈。

\section{3 人迹地图平台}

人迹地图平台（图 7, 图 8) 作为衡复空间分析模块, 旨 在实现微城市空间的分析和中观不同片区的对比。平台目前

\section{表 3 街道慢行指数测度数据接口}

\begin{tabular}{|c|c|c|}
\hline 慢行指数维度 & 具体指标 & 数据接口 \\
\hline \multirow{4}{*}{ 吸引力 } & 到最近设施距离 & 路网数据、服务业态点数据 \\
\hline & 用地混合度 & 路网数据、各类用地功能点数据 \\
\hline & 微博心情指数 & 微博與情数据 \\
\hline & 底商密度 & 商业点数据 \\
\hline \multirow{6}{*}{ 安全性 } & 拥堵程度 & 地图实时拥堵数据 \\
\hline & 人行道宽度比例 & \multirow{4}{*}{ 路网数据 } \\
\hline & 自行车道比例 & \\
\hline & 路网密度 & \\
\hline & 空间句法整合度 & \\
\hline & 街道人行空间 & 街景图片 \\
\hline \multirow{6}{*}{ 舒适性 } & 遮阴率 & \multirow{6}{*}{ 街景图片 } \\
\hline & 绿地空间 & \\
\hline & 自行车空间 & \\
\hline & 街道卫生 & \\
\hline & 街道景观 & \\
\hline & 机动车停车问题 & \\
\hline \multirow{4}{*}{ 历史性 } & 建筑 & \multirow{4}{*}{ 街景图片 } \\
\hline & 小型开放空间 & \\
\hline & 城市空间节点 & \\
\hline & 街坊内公共步行交通 & \\
\hline
\end{tabular}

\section{表 4 人本观测平台功能模块、数据接口和传感器}

\begin{tabular}{l|l|c}
\hline \multicolumn{1}{c|}{ 功能模块 } & 数据接口 & 传感器 \\
\hline $\begin{array}{l}\text { 实时监测街区的访客流量, 并按日、时显示变化 } \\
\text { 曲线 }\end{array}$ & 人流数据 & $\begin{array}{c}\text { 场地卡口 } \\
\text { 感应设施 }\end{array}$ \\
\hline $\begin{array}{l}\text { 识别观测地区的访客群体, 根据已有的手机 APP 数 } \\
\text { 据分析访客属性,显示珫计结果。访客属性包括性别、 } \\
\text { 年龄、婚否、是否有车、手机品牌和所在省份等 }\end{array}$ & WIFI 探针 \\
\hline $\begin{array}{l}\text { 通过定点布设的摄像头监测停车位使用情况, 按小 } \\
\text { 时在平台上显示统计结果 }\end{array}$ & 停车数据 & 摄像头 \\
\hline $\begin{array}{l}\text { 周期性地 (按月或季度) 采集观测地区发布的新浪 } \\
\text { 微博并分析显示出相关微博的关键词, 作为與情信料 }\end{array}$ & $\begin{array}{l}\text { 数据 } \\
\text { 息的反映 }\end{array}$ & - \\
\hline
\end{tabular}

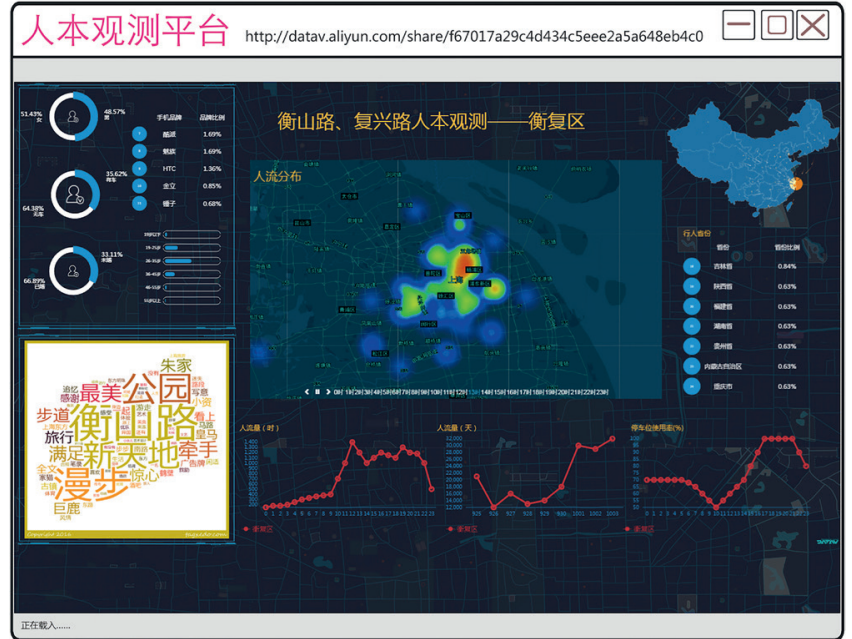

图 6 衡复人本观测平台

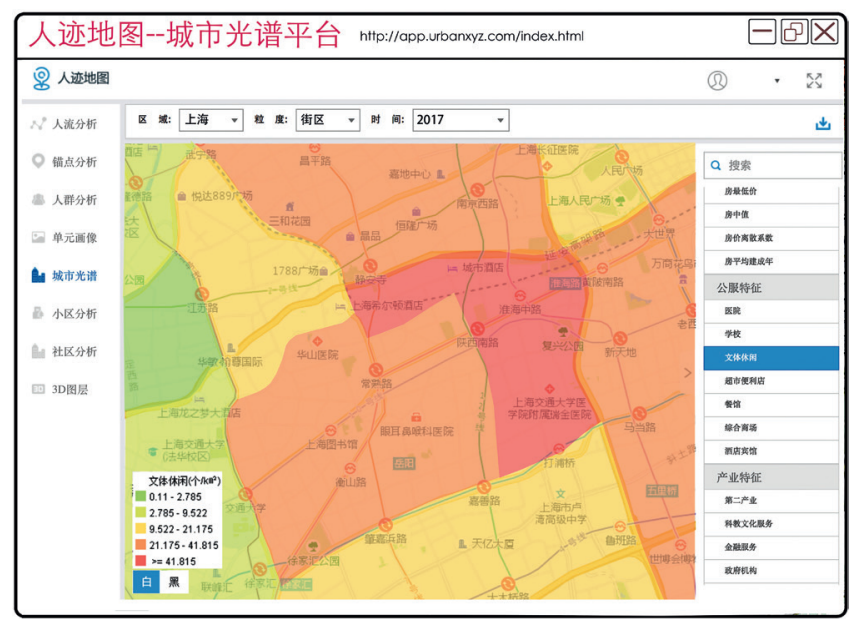

图 7 人迹地图平台一一城市光谱

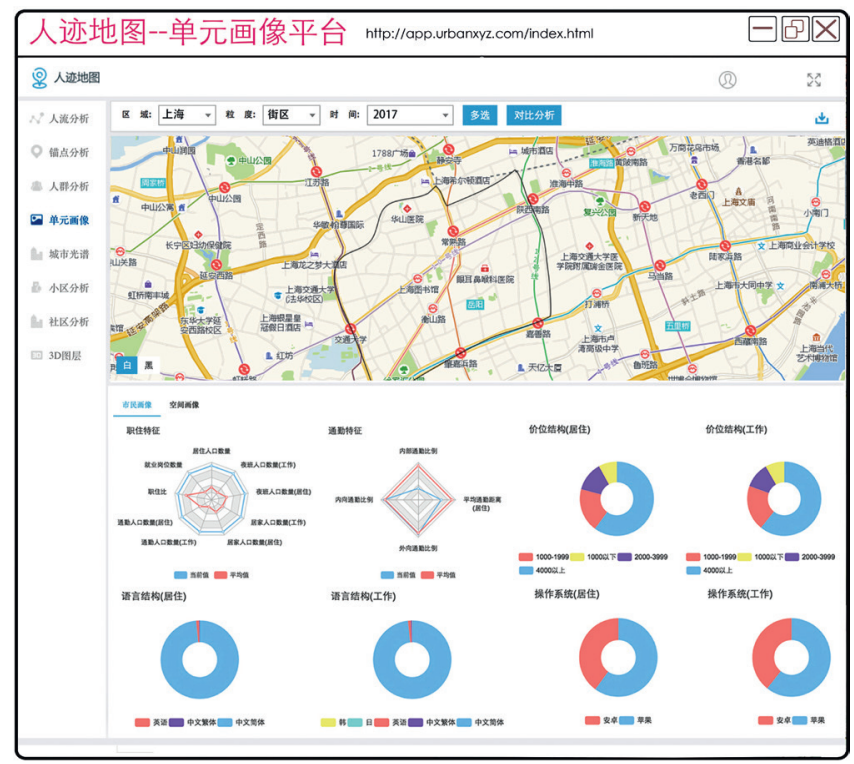

图 8 人迹地图平台一单元画像 
的主要功能分为城市光谱和单元画像两大模块。其中核心模 块为城市光谱, 包含城市交通、房价、公服设施 (POI)、产 业等四个类别 17 项指标, 以衡复地区现有的研究地块边界 为空间单元, 进行各项指标的整合和统计。单元画像模块则 显示每个空间单元的数据与整体平均值的对比。人迹地图模 块主要为互联网的开放数据, 更新周期为一年左右。长期使 用该平台, 可实现衡复历史街区与规划设计相关的多源背景 数据的记录与分析, 作为 $\mathrm{A} / \mathrm{B}$ 类街道重新划分和整体设计 策略调整的提供基础。

\section{4 方案宣传与展示平台}

方案宣传展示平台（图 9）受众主要为城市管理者、规 划人员、市民、开发商等多类利益相关者, 可使公众更加直
观地了解规划设计方案, 增强对于规划的参与感和认同感, 奠定公众参与的基础。

\section{5 公众参与平台}

公众参与平台（图 10）旨在促使利益相关者提出不同 的设计意见, 对方案实施进行评价, 并促进多元的沟通与协 商; 同时根据公众反馈的意见, 结合多时态微观数据对规划 设计进行动态调整。在表达对设计方案的意见时, 公众可通 过操作简易的交互式界面, 对基地内各项设施作出增减调整, 对多种社区的更新模式等进行选择；同时居民也可选择偏好 的城市空间意向, 作为 $\mathrm{A} / \mathrm{B}$ 类街道测度指标权重计算的依据。 公众参与平台既充分尊重居民的意愿, 又避免了由于公众专 业性知识的缺乏导致的决策效率低下问题。

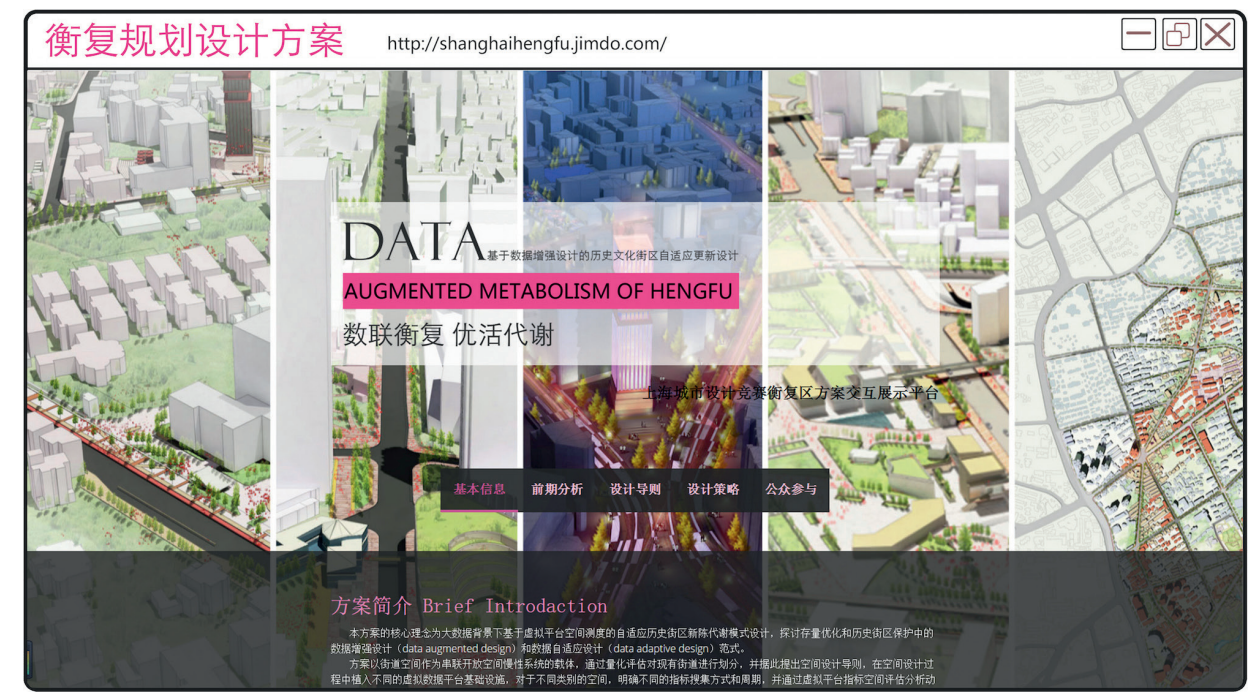

图 9 衡复方案宣传与展示平台

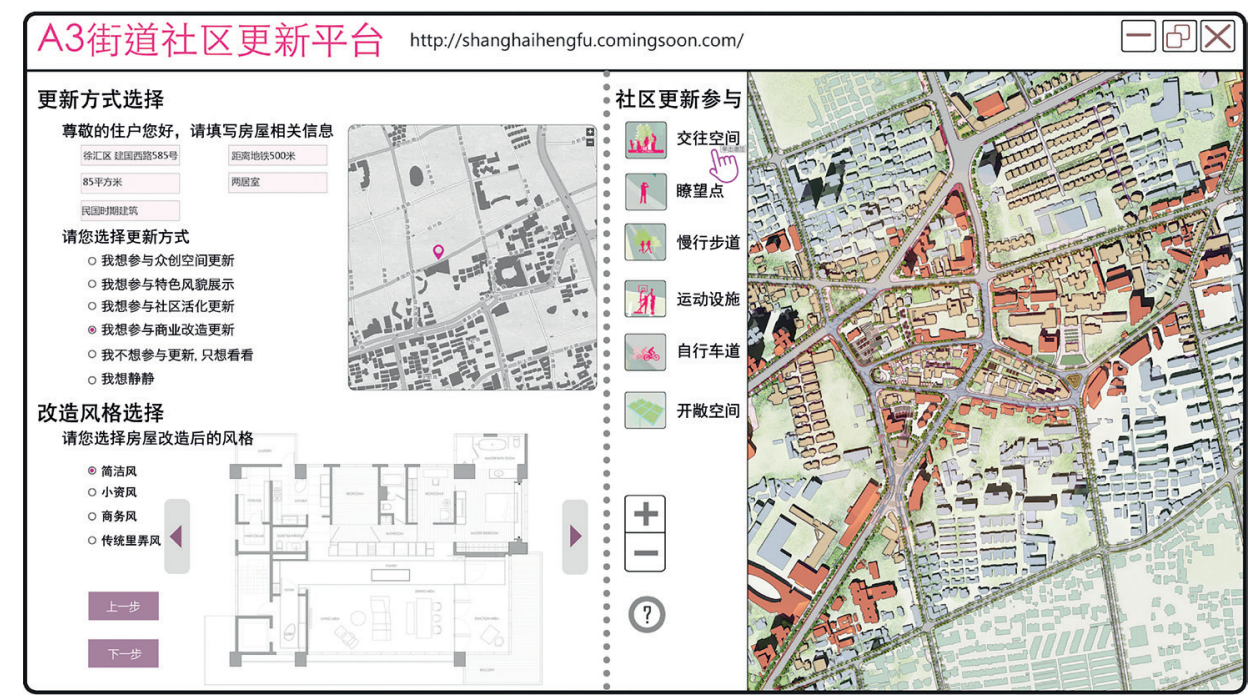

图 10 公众参与平台 


\section{6 人际数据交互感知地毯}

此外, 方案创新地提出了人迹数据交互感知地毯装置 (图 11)。衡山路一乌鲁木齐南路的数据观光塔为衡复新地 标, 其下的广场与邻近街道铺设结合灯槽照明的人际数据交 互感知地毯, 动态搜集人流与车流的多种数据, 并能反馈到 塔立面镶嵌的交互照明装置中, 人流越大, 塔身越亮, 使得 人们即使在公里之外也能感受到衡复中心的活力。这种数据 交互与可视化的装置为人们感知城市提供了新的体验, 人们 可以根据交互界面的反馈动态设计自己的行为路线等。

\section{3 总结与讨论}

本文在回溯数据增强设计应用的基础上, 提出数据在规 划设计后置式反馈的重要性, 构建了基于传感器基础设施和 在线平台的自反馈城市设计方法, 这一方法是对数据增强设 计理念的延续。本文对该方法的整体架构、数据类型、传感 器类型、平台框架和应用场景进行了详细的阐述, 最后将这 一自反馈城市设计的方法初步运用于上海衡复历史街区的城 市设计实践中, 以衡复信息共享交互平台为例进行说明。

传统的城市规划设计经历了蓝图式规划向多元价值融合 和多元主体参与导向的转变, 大数据的出现使得城市规划与 设计者在方案的形成期得以掌握更加充分、精细化的信息, 对抽象的空间状态和人群行为进行精细化度量, 从而辅助方
案的生成, 但由于大数据不开放, 或开放数据过于稀疏、有 偏等问题, 现有数据类型对方案实施后的动态追踪显得乏力。 本文正是基于城市传感器和在线平台的运用潜力, 创新性地 提出了数据针对后置式反馈城市设计的应用模式。

自反馈的城市设计以设计方案为导向建立空间评估框架 和指标, 在空间中布置传感器, 从而开展对人群对象的动态 追踪和空间环境的精准测度。通过提高传感器的布置密度提 升数据的精度, 通过缩短数据搜集的周期提高自反馈的频率, 甚至实现实时反馈与空间诱导的目标。在线平台经过数据处 理、综合分析、特征提取和指标可视化后, 形成了开放互动 的公共展示界面。不仅规划管理者可以评估方案的实施进行 空间干预、设施优化、需求匹配、实时调控; 而且民众可以 获取空间信息实现出行的优化, 了解规划动态, 从而促进公 众参与。

诚然, 传感器的自定义和在线平台开发存在成本较高和 技术困难的限制, 同时传感器在现实空间中安装也存在与城 市法规与管理冲突的问题。如何有效解决传感器和现有城市 空间的良性融合, 构建精简、有效、融合的在线平台仍需要 不断的实践, 并对架构进行优化。此外, 城市规划和设计方 案由于类型和地区的多样性, 将存在对城市传感器各种各样 的需求, 彼此存在重叠或冲突, 如何建立统一的传感器布置 规范, 实现传感器的优化配置也是未来需要探讨的问题。

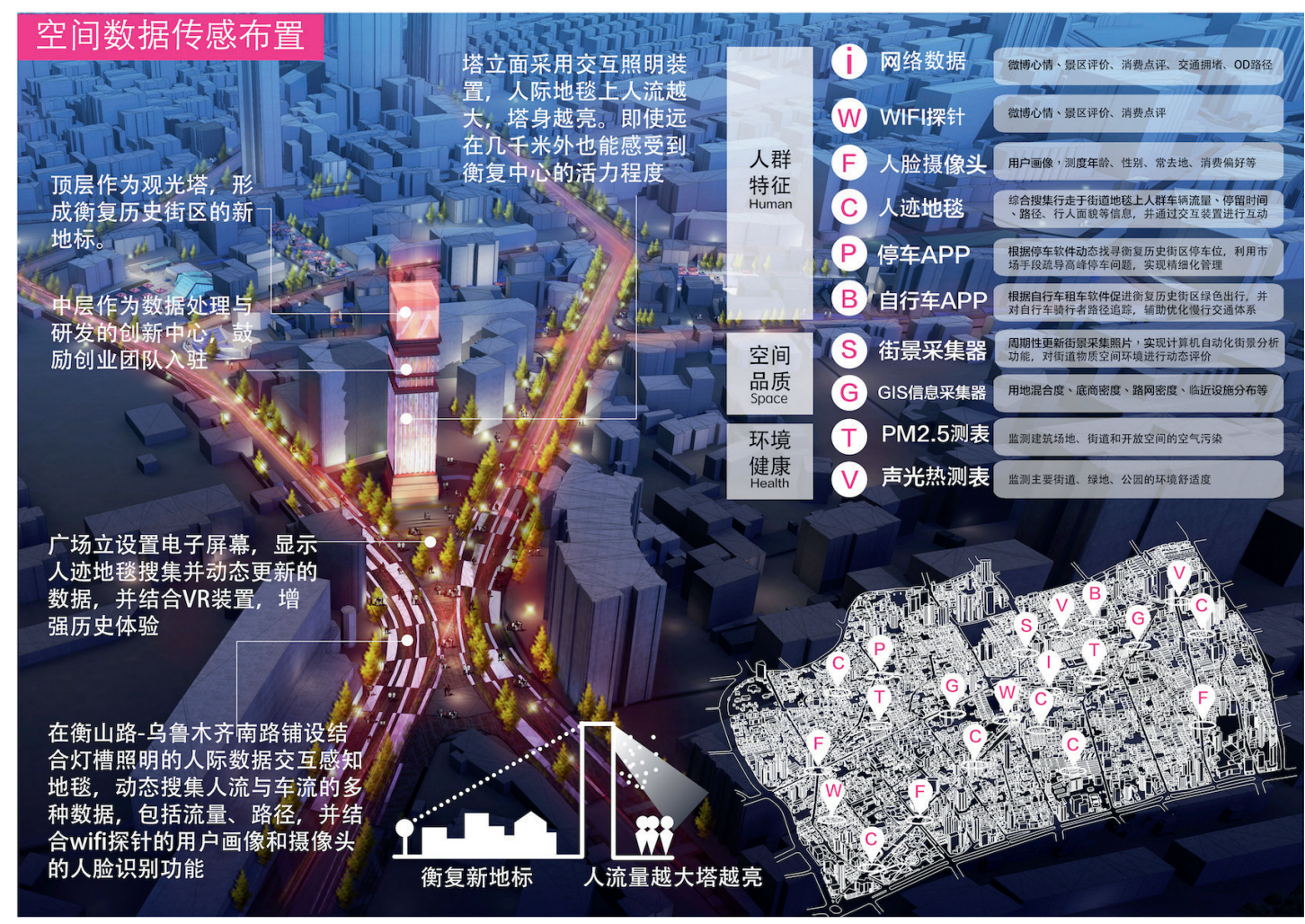

图 11 衡复空间传感器布置 
本研究是对传感器和在线平台支撑的后置式自反馈城市 设计的初步探索, 未来将进一步扩展运用场景; 针对不同的 场景丰富传感器类型、数据类型和在线平台的综合架构; 进 行传感器搭建和在线平台反馈的真实模拟实验, 探索传感器 与真实空间融合并实现人群交互的设计方法。UPI

注: 文中图表均为作者绘制。

感谢北京市城市规划设计研究院的茅明睿和姜冬睿对本文 案例中平台开发实现的技术支持。

\section{参考文献}

[1] 龙瀛, 沈尧. 数据增强设计一一新数据环境下的规划设计回应与改变 [J]. 上海城市规划, 2015(2): 81-87.

[2] 龙瀛. 数据增强设计最新研究进展及其教学实践 [J]. 理想空间, 2016(8): 04-07.

[3] 甘欣悅, 龙瀛. 新数据环境下的量化案例借鉴方法及其规划设计 应用 [J/OL]. 国际城市规划. (2017-07-20). http://kns.cnki.net/kcms/ detail/11.5583.tu.20170720.1115.001.html.

[4] 曹哲静, 龙瀛. 数据自适应城市设计的方法与实践——上海衡复历 史街区慢行系统设计为例 [J]. 城市规划学刊, 2017(4): 47-55.

[5]盛强。“数据游骑兵”实用战术解析: 空间向法在短期城市设计工作营 设计教学中的应用 [J]. 时代建筑, 2016(2): 140-145.

[6] 徐否青, 马晨, 周峰, 等.轨交站域的人流、商业与城市设计一以上 海“静安寺站”和“中山公园站”为例 [J]. 建筑学报, 2015(S1): 60-65.

[7] 储妍, 茅明蓂, 高硕, 等. 数据如何驱动设计——回龙观社区品质提 升为例 [J]. 规划师, 2017, 33(2): 81-89.

[8] 茅明禀, 储奸, 张鹏英, 等. 人迹地图: 数据增强设计的支持平台 [J]. 上海城市规划, 2016(3): 22-29, 89.

[9] 杨俊宴, 曹俊. 动. 静. 显. 急: 大数据在城市设计中的四种应用模式 [J]. 城市规划学刊, 2017(4): 39-46.

[10] 龙瀛, 叶宇. 人本尺度城市形态: 测度、效应评估及规划设计响应 [J]. 南方建筑, 2016(5): 39-45.

[11] 龙瀛，沈尧.大尺度城市设计的时间、空间与人 (TSP) 模型——突破 尺度与粒度的折中 [J]. 城市建筑, 2016(6): 33-37.

[12] 周榕, 杜頔康. 周榕: 互联网是新的城市, 城市就是曾经的乡村 [J]. 住区, 2017(1): 114-119.

[13] BATTY Michael. Big data, smart cities and city planning. Dialogues in Human Geography 3.3 (2013): 274-279.

[14] BATTY Michael. Urban informatics and big data. ESRC (The UK Economic and Social Research Council) Cities Expert Group, 2013.

[15] MAZHAR M Rathore, AWAIS Ahmad, ANAND Paul, et al. Urban planning and building smart cities based on the internet of things using big data analytics[J]. Computer Networks, 2016, 101: 63-80.

[16] VAKALI Athena, ANTHOPOULOS Leonidas, KRCO Srdjan. Smart cities data streams integration: experimenting with internet of things and social data flows $[\mathrm{C}]$. Proceedings of the 4th International Conference on Web Intelligence, Mining and Semantics (WIMS14), 2014: 60.

[17] FRENCH Steve, BARCHERS Camille, ZHANG Wenwen. Moving beyond operations: leveraging big data for urban planning decisions[C]. 56th Annual Conference of Association of College Schools of Planning (ACSP), Portland, OR, 2015. 